\title{
Editorial
}

\section{Assessing food intake through a chest-worn camera device}

Over the past decade, new technological and conceptual advances have opened innovative avenues for dietary assessment. Two basic conceptual notions can be distinguished. 'New methodologies' include distinct approaches of collecting dietary information (e.g. integrating and combining different types of self-reported methods and/or biomarkers or metabolomics) while 'new technologies' relate to new or improved ways to collect, handle, disseminate, exchange and analyse dietary data (e.g. mobile phones). Prevailing misconceptions about these concepts obscure the understanding and proper evaluation and use of these new applications.

Illner et al. ${ }^{(1)}$ recently reviewed emerging technologies and methodologies and their relative merits for improving dietary assessment. Particular attention was given to their potential to replace, improve or complement conventional self-reported dietary methodologies such as FFQ, $24 \mathrm{~h}$ dietary recalls and food records. The review applied a series of predefined evaluation criteria for this purpose. A main conclusion of the review was that interactive computer- and web-based technologies' were found to be good innovative alternatives to conventional FFQ or $24 \mathrm{~h}$ dietary recalls, whereas 'personal digital assistant, mobilephone- or camera- and tape-recorder-based technologies' were considered good alternatives or complements to paper-based food records. Clearly, the definitions, classifications and evaluation criteria will require regular revisions to reflect the extremely dynamic development of new dietary technologies.

In this issue of Public Health Nutrition, Jia et al. ${ }^{(2)}$ present a small chest-worn electronic recording device to assess foods and estimate food volumes. The so-called 'e-Button' device automatically takes two-dimensional pictures of foods every 2 to $4 \mathrm{~s}$ and matches those images against a geometric-shape library. Subjects attach the device to their clothing using a pair of magnets or a pin and only need to turn on and off a button on the device to activate the operating status before an eating occasion. Images are modelled virtually and manipulated by means of a complex mathematical model ${ }^{(3)}$ in order to calculate the volume of food consumed from the two-dimensional image. To evaluate the accuracy of the 'e-Button', Jia et al. ${ }^{(2)}$ compared the computer-calculated food volumes with the actual three-dimensional volumes determined by the seed displacement method as a gold standard. The results revealed that the computer-calculated estimates show less bias and variability than the visually estimated food volumes.
A prototype for the 'e-Button',(4) contained several sensors and data processing and storage elements and was worn on a lanyard around the neck. However, due to its new methodological nature, its strengths and weaknesses were not evaluated relative to those of conventional dietary assessment methods.

Table 1 summarises the main strengths and weaknesses of the device according to the evaluation criteria used in the earlier review ${ }^{(1)}$. Overall, the 'e-Button' device can be considered a promising new methodology opening many new opportunities. Both the automatic and immediate recording of digital pictures during eating occasions and the semi-automatic calculation of food volumes contribute to good overall identification and visualisation of the amounts of many foods and recipes consumed (i.e. those that are consumed from a plate), independently from the subject's participation. As this new methodology is unable to quantify all foods consumed, it should be seen more as a complementary rather than a stand-alone methodology, at least in the context of large nutritional studies. However, it could be of great interest as a reference method for validation/calibration, or for nutritional intervention/clinical studies conducted in controlled conditions, with relatively smaller sample sizes and with one or limited study site(s), as compared with observational research settings.

To be considered a more complete and accurate dietary assessment, the 'e-Button' device should move towards assessing more detailed information on a more complete set of foods consumed and/or provide insights on how this approach could be better integrated/complemented with other, more traditional methodologies for a holistic measurement of the whole individual diet. Currently, the 'e-Button' device provides only a snapshot of food consumption in time, and estimation of individual usual intake requires repeated (non-consecutive) day assessments. Further research on the capacity of the device to handle all the pictures over a whole day period is required. In addition, more insight is needed on the reproducibility, validity and standardisation of the device within and between individuals and types of foods considered. The cost-effectiveness of the 'e-Button' at different steps of the process, including technical and training investments, is also needed for a better evaluation of this new methodology.

There are many other challenges to be considered when using the 'e-Button' device for research purposes. The authors reported in the present and former papers on the difficulties of determining accurate portion sizes of 
Table 1 Strengths and weaknesses of the 'e-Button' device

\begin{tabular}{ll}
\hline Strengths & Weaknesses (comments) \\
\hline - Semi-automatic calculation of food & - So far, assessment of a snapshot of food consumption in time (The methodology \\
volumes based on real quality-controlled & requires foods to be available from a plate. It is not clear how the new method will \\
pictures, independently from the study & integrate estimates of all other foods consumed (e.g. foods consumed not from a \\
participants & plate, liquids, non-visible ingredients)) \\
- Memory-independent, immediate & - Certain foods cannot be correctly identified from the images (Indeed, the 'e-Button' \\
recording, low likelihood to recall bias & is currently unable to correctly identify/quantify all foods consumed (e.g. lack of \\
- Good overall identification and & identification of added foods, non-visible foods/ingredients or complex dishes (e.g. \\
visualisation of foods/recipe consumed & fat, salt), beverages, foods consumed not from a plate) and cooking methods. This \\
- Moderate to good accuracy of portion size & requires to be complemented by asking additional questions of the subjects or by \\
quantification (compared with seed & (conventional) approaches to collect missing dietary information. This will increase \\
displacement method) & logistical efforts for the researcher and burden to the study subjects. Although the \\
- Low training efforts, low cognitive efforts & method should reduce recall bias, changes in individuals' usual habits cannot \\
for completion, no requirement for literacy & completely be disregarded and participants could switch off the 'e-Button' for foods \\
or computer skills & they do not want to be recorded \\
& $\begin{array}{l}\text { Conversion to individual food intake is difficult if food items cannot be identified and } \\
\text { classified }\end{array}$ \\
- Blurred pictures, unfavourable observation angle, lighting environment, complex \\
food shapes can hamper accurate food volume estimation ('e-Button' has an
\end{tabular}

a number of specific foods, such as stewed pork and potato, and of irregularly shaped foods such as ice cream. Incorrect positioning of the observation angle, poor operation in areas of low light and irregular food shapes unmatchable with items in the data library are some possible sources of measurement error identified by the authors. Variability in subject compliance and ethical and privacy concerns may be further issues. In addition, retrospective review of 'e-Button' device images by the wearer might change eating behaviour - although this suggests the potential of the device to be used in nutritional promotion studies, e.g. of fruit and vegetable consumption.

In conclusion, this new methodology benefiting from advanced technologies is very promising for better identification and quantification of foods consumed. However, several challenges still need to be addressed for full evaluation of the reproducibility, validity and standardisation of this new approach. In addition, more insights should be provided on its cost-effectiveness and integration with other existing (more traditional) approaches for a more holistic measurement of individual intakes.
Anne-Kathrin Illner and Nadia Slimani Dietary Exposure Assessment Group (DEX) Nutrition and Metabolism Section, International Agency for Research on Cancer/Word Health Organization Lyon, France Email: IllnerA@fellows.iarc.fr

Carl Lachat Deputy Editor Email: carl.lachat@ugent.be

\section{References}

1. Illner AK, Freisling H, Boeing $\mathrm{H}$ et al. (2012) Review and evaluation of innovative technologies for measuring diet in nutritional epidemiology. Int J Epidemiol 41, 1187-1203.

2. Jia W, Chen H-C, Yue Y et al. (2014) Accuracy of food portion size estimation from digital pictures acquired by a chest-worn camera. Public Health Nutr 17, 1671-1681.

3. Chen HC, Jia W, Yue Y et al. (2013) Model-based measurement of food portion size for image-based dietary-assessment using 3D/2D registration. Meas Sci Technol 24, 105701.

4. Sun M, Fernstrom JD, Jia W et al. (2010) A wearable electronic system for objective dietary assessment. J Am Diet Assoc 110, 45-47. 